\title{
Severe acute respiratory syndrome: Did quarantine help?
}

\author{
Richard Schabas MD MHSc FRCPC
}

Q uarantine, the isolation of asymptomatic individuals who are thought to be incubating infection, was a prominent control strategy used in the recent severe acute respiratory syndrome (SARS) outbreaks. A recent report about the public health efforts to control SARS in Toronto concluded that in future outbreaks "for every case of SARS, health authorities should expect to quarantine up to 100 contacts" (1).

This is a remarkable conclusion. It is one thing to resort to an unproven intervention in the crisis posed by a novel disease threat; however, it is quite another to recommend the continued use of this intervention after the dust has settled and we know, or should know, a great deal more about the problem at hand. Mass quarantine for disease control was essentially abandoned last century. Does it deserve a second look?

An outbreak should meet the following three criteria for quarantine to be a useful measure of disease control:

- first, people likely to be incubating the infection must be efficiently and effectively identified;

- second, those people must comply with the conditions of quarantine, and;

- third, the infectious disease in question must be transmissible in its presymptomatic or early symptomatic stages.

The use of quarantine in the Toronto outbreak failed on all three counts.

SARS quarantine in Toronto was both inefficient and ineffective. It was massive in scale. Toronto public health authorities quarantined approximately 100 people for each SARS case, while Beijing public health quarantined about 12 people for each SARS case. An analysis of the efficiency of quarantine in the Beijing outbreak conducted by the American Centers for Disease Control and Prevention concluded that quarantine could have been reduced by two-thirds, (four people per SARS case) without compromising effectiveness if authorities had "focused only on persons who had contact with an actively ill SARS patient" (2).

This analysis suggests that Toronto quarantined at least 25 times more people than was appropriate. Concerns about this inefficiency were raised quite early in the outbreak $(3,4)$.

The Toronto quarantine was clearly ineffective in identifying potential SARS patients. At least the first 50 cases in the second phase of the outbreak were not quarantined.

Compliance with the Toronto quarantine was poor. Only $57 \%(13,291$ of 23,103$)$ of people quarantined were 'compliant', according to Toronto officials (1), although how this was defined and measured is not clear. It is hard to understand how anyone could attribute the rapid and effective elimination of an infectious disease to an intervention with such low compliance.

We now know a great deal more about the natural history of SARS and its transmission. In fact, the evidence is compelling and shows that SARS is not infectious during the preclinical phase and does not become significantly infectious until the symptomatic illness is well-established. Peak infectivity is in the second week of clinical illness (5). If ever an infectious disease was ill-suited for quarantine, it is SARS.

Did quarantine work for SARS? Notwithstanding the conclusions of the Toronto public health group, I think the evidence is now overwhelming that quarantine played little or no role in controlling SARS. Furthermore, mass quarantine, as practiced in Toronto, did considerable harm by sapping public health resources and fueling public anxiety.

SARS was rapidly controlled and eradicated in Toronto and everywhere else that it appeared. Fundamentally, this is because SARS is only capable of sustained transmission in hospitals that do not suspect its presence. SARS is not capable of sustained transmission in the community (6). Case identification and isolation in hospitals is what controlled SARS. Quarantine, as such, played no role.

In the unlikely event of another SARS outbreak in Canada, public health officials should quarantine no one. Instead, they should identify and observe close contacts of cases, ie, people with a 'reasonable suspicion' of SARS. These close contacts should be isolated if, and only if, they develop symptoms consistent with the current recommendations of the World Health Organization (5).

\section{REFERENCES}

1. Svoboda T, Henry B, Shulman L, et al. Public health measures to control the spread of severe acute respiratory syndrome during the outbreak in Toronto. N Engl J Med 2004;350:2352-61.

2. Efficiency of quarantine during an epidemic of severe acute respiratory syndrome - Beijing, China. MMWR Morb Mortal Wkly Rep 2003;52:1037-40.

3. Dwosh H, Hong H, Austgarden D, Herman S, Schabas R. Identification and containment of an outbreak of SARS in a community hospital. CMAJ 2003;168:1415-20.

4. Schabas R. SARS: Prudence, not panic. CMAJ 2003;168:1432-4.

5. World Health Organization. Consensus document on the epidemiology of severe acute respiratory syndrome (SARS). WHO/CDS/CSR/GAR/ 2003;11:4, 8-10.

$<$ www.who.int/csr/sars/en/WHOconsensus.pdf > (Version current at July 22, 2004).

6. Low D. Why SARS will not return: A polemic. CMAJ 2004;170:68-9. 


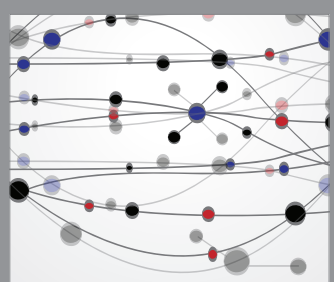

The Scientific World Journal
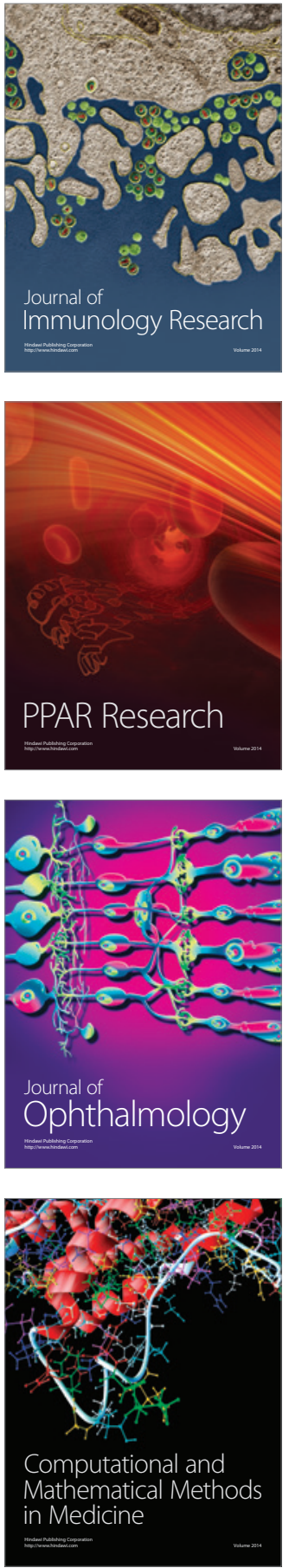

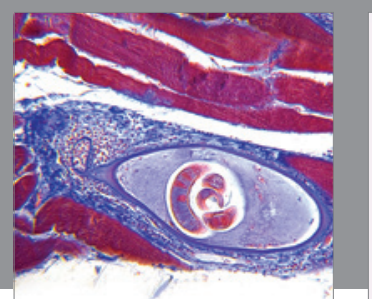

Gastroenterology Research and Practice

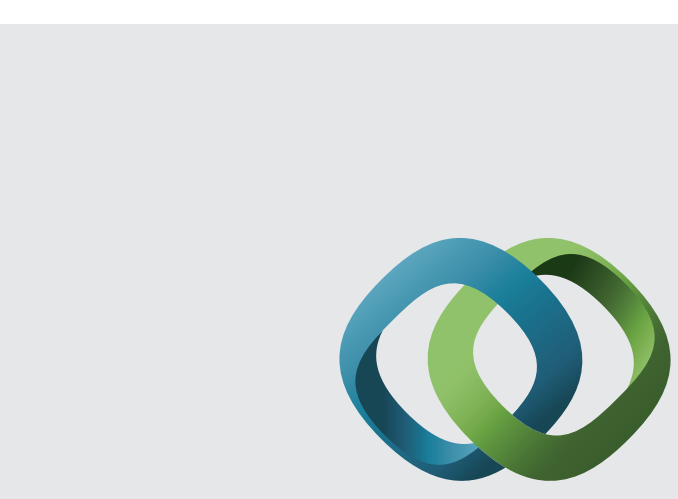

\section{Hindawi}

Submit your manuscripts at

http://www.hindawi.com
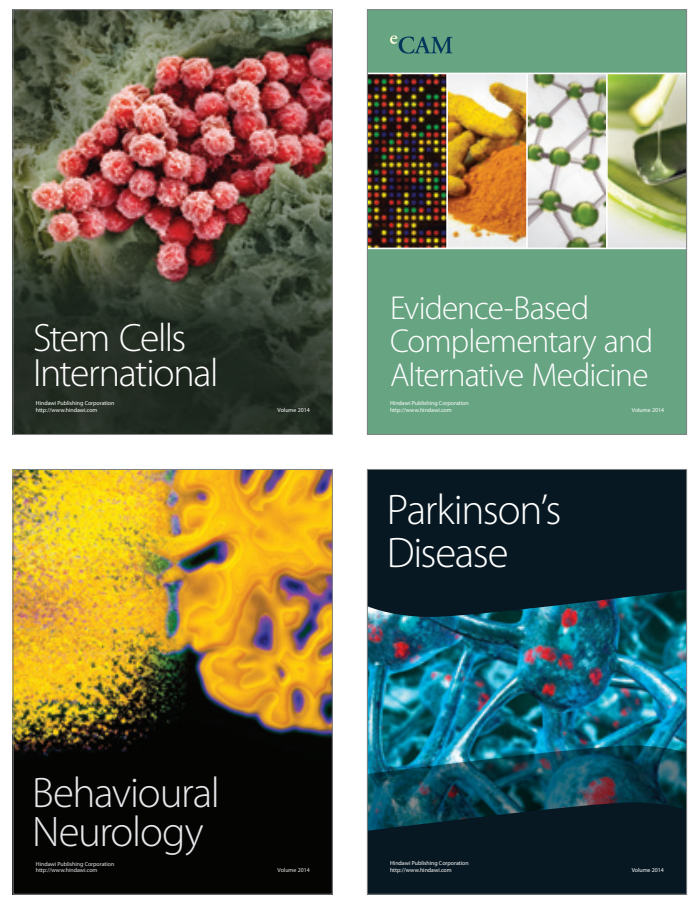
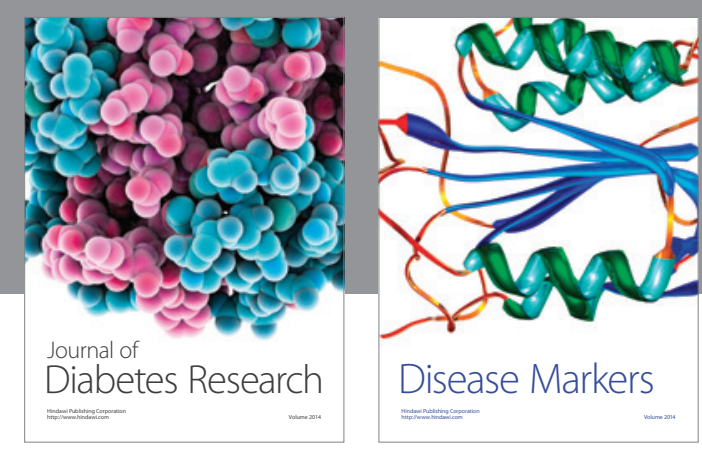

Disease Markers
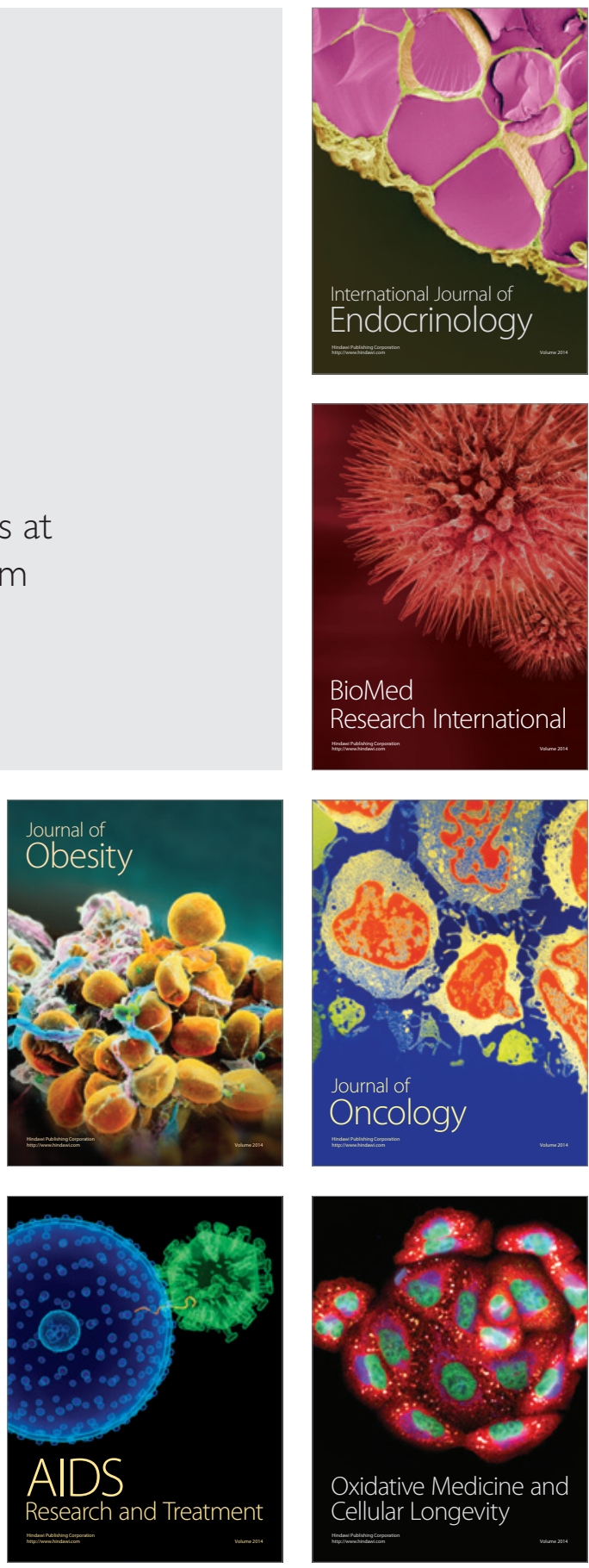